\title{
29 ELEVATED FLT3L PREDICTS LONG-TERM SURVIVAL IN PATIENTS WITH HIGH-GRADE GASTROENTEROPANCREATIC NEUROENDOCRINE NEOPLASMS
}

${ }^{1}$ Katharina Detjen, ${ }^{2}$ Raik Otto, ${ }^{1}$ Yvonne Giesecke, 'Lukas Geisler, ${ }^{1}$ Pamela Riemer, ${ }^{1}$ Henning Jann, ${ }^{1}$ Carsten Grötzinger, ${ }^{1}$ Christine Sers, ${ }^{3}$ Tom Luedde, ${ }^{2}$ Ulf Leser, ${ }^{1}$ Bertram Wiedenmann, ${ }^{1}$ Michael Sigal, ${ }^{1}$ Frank Tacke, ${ }^{3}$ Christoph Roderburg, ${ }^{1}$ Linda Hammerich*. 'Charité Universitätsmedizin Berlin, Berlin, Germany; ${ }^{2}$ HumboldtUniversität zu Berlin, Berlin, Germany; ${ }^{3}$ University Hospital Düsseldorf, Duesseldorf, Germany

Background Gastroenteropancreatic neuroendocrine neoplasms (GEP-NEN) are a rare and heterogeneous family of tumors arising from the disseminated neuroendocrine system of the gastrointestinal tract and pancreas. Clinical management of high-grade GEP-NEN is challenging due to disease heterogeneity, illustrating the need for reliable biomarkers facilitating patient stratification and guiding treatment decisions. FMS-like tyrosine kinase 3 ligand (Flt3L) is emerging as a prognostic or predictive surrogate marker of host tumoral immune response and might enable stratification of patients with otherwise comparable tumor features.

Methods We used RNAseq data from human foregut-derived pancreatic and gastric GEP-NEN to evaluate Flt3L gene expression in tumor tissue. The data set $(n=54)$ represented the full range of NEN grades and differentiation, and expression levels were compared to healthy control tissue as well. We also analyzed circulating Flt3L levels in serum samples of a separate cohort of G2/G3 GEP-NEN $(n=59)$ an healthy controls $(n=4)$. The study was approved by the local ethics committee at Charité Universitätsmedizin Berlin, Germany (ethical approval number EA1/229/17) and patient informed consent was obtained.

Results We detected a prominent induction of Flt3L gene expression in individual G2 and G3 NEN, but not in G1 neuroendocrine tumors (NET). Flt3L mRNA expression levels in tumor tissue predicted disease related survival of patients with highly proliferative G2 and G3 NEN more accurately than the conventional criteria of grading or NEC/NET differentiation. High level Flt3L mRNA expression was associated with increased expression of genes related to immunogenic cell death, lymphocyte effector function and dendritic cell maturation, suggesting a less tolerogenic (more proinflammatory) phenotype of tumors with Flt3 $\mathrm{L}$ induction. Importantly, circulating levels of Flt3L were also elevated in high grade NEN and correlated with patients' progression-free and diseaserelated survival, thereby reflecting the results observed in tumor tissue.

Conclusions Our results suggest Flt3L as a surrogate marker of an inflammatory tumor microenvironment. Therefore, we propose Flt3 $\mathrm{L}$ as a prognostic biomarker for high grade GEPNEN. Flt3L measurements in serum, which can be easily be incorporated into clinical routine, may hold the promise to guide patient stratification and tailor treatment decisions and should be further evaluated, especially in the context of immunotherapies.

Ethics Approval The study was approved by the local ethics committee at Charité Universitätsmedizin Berlin, Germany (ethical approval number EA1/229/17) and patient informed consent was obtained.

http://dx.doi.org/10.1136/jitc-2021-SITC2021.029 